\title{
Ground Penetrating Radar Assessment of Flexible Road Pavement Degradation
}

\author{
Sandro Colagrande, ${ }^{1}$ Danilo Ranalli, ${ }^{2}$ and Marco Tallini ${ }^{2}$ \\ ${ }^{1}$ Dipartimento di Architettura e Urbanistica, Università di L'Aquila, Via Campo di Pile, Zona industriale di Pile, 67100 Aquila, Italy \\ ${ }^{2}$ Dipartimento di Ingegneria delle Strutture, delle Acque e del Terreno, Università di L'Aquila, Nucleo Industriale di Bazzano, \\ Monticchio 67100 Aquila, Italy
}

Correspondence should be addressed to Marco Tallini, marco.tallini@univaq.it

Received 7 July 2010; Revised 27 October 2010; Accepted 8 February 2011

Academic Editor: Mrinal Sen

Copyright (c) 2011 Sandro Colagrande et al. This is an open access article distributed under the Creative Commons Attribution License, which permits unrestricted use, distribution, and reproduction in any medium, provided the original work is properly cited.

GPR investigations were used to study degraded road pavements built in cutting sections. Road integrity was assessed via quantitative analysis of power curves. $1600 \mathrm{MHz}$ and $600 \mathrm{MHz}$ radar sections were collected in 40 damaged and undamaged road pavement sites. The collected data were processed as follows: (i) linearisation with regression analysis of power curves; (ii) assessment of absorption angle $\alpha^{\prime}$ which is directly proportional to absorption coefficient $\alpha$ (this was obtained by setting the e.m. propagation velocity to $10 \mathrm{~cm} / \mathrm{ns}$ ); (iii) comparison of absorption coefficients in both damaged and undamaged zones with respect to road pavement degradation. If the absorption coefficients of damaged and undamaged road sections have nearly the same value, then the likely cause of degradation is the fatigue or the thermal shrinkage; if they are not, then road degradation is due to the different compactness of the soil caused by vehicular traffic load. In a considerable number of sites, the statistical comparison of damaged and undamaged zones through the absorption coefficient analysis shows that surface observations of road pavements are quite consistent with power curve analyses.

\section{Introduction}

Defects in flexible pavements are indicative of road distress and impaired efficiency. They may be due to poor performance of constituent materials, errors in design, engineering or construction and environmental and climate factors as well as to particularly heavy traffic. Two types of defects may be distinguished: functional and structural [1].

Functional defects express shallow degradation of the wearing course of pavements; this degradation reduces both vehicle grip to the road and evenness of the road surface, jeopardising traffic safety. Factors responsible for this type of degradation and giving rise to skid resistance problems include levelling (or polishing) of aggregates; surface exposure of bitumen (known as bitumen blooming); disgregation and detachment of aggregates. Smoothness problems comprise longitudinal undulations, transverse undulations (more commonly called ruts); hollows or bulges; dips on extensive surfaces; edge cracking.
Conversely, structural defects arise in the supporting courses of the superstructure. They are due to deterioration of its load-bearing capacity and have major repercussions on pavement durability, if they are not timely cured. Defects of this type encompass surface cracks and breaks and, more recurrently, longitudinal, and transverse cracks, longitudinal cracks only, transverse cracks only, ramified cracks (spider or alligator cracks), and failures [2-4].

The GPR study was focused on road pavements with structural defects compromising their load-bearing capacity (Figure 1).

Now, it is worth reviewing the potential factors which trigger structural degradation of flexible road pavements. These factors may be related both to problems occurring upon construction and to effects supervening during road use. In particular, permanent deformation of the subgrade and of the loose and bound granular materials making up the pavement (taking place during construction) may result from a combination of different (and usually concurrent) 


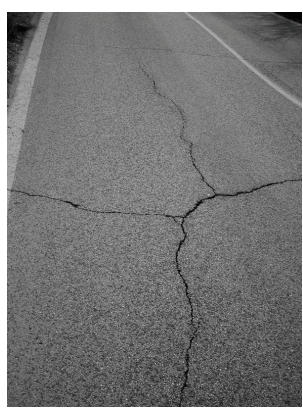

(a)

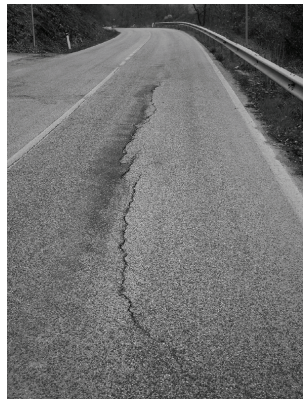

(b)

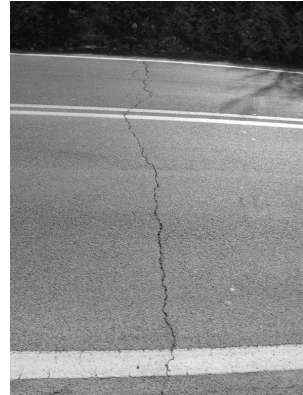

(c)

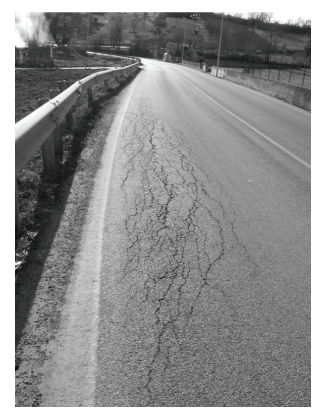

(d)

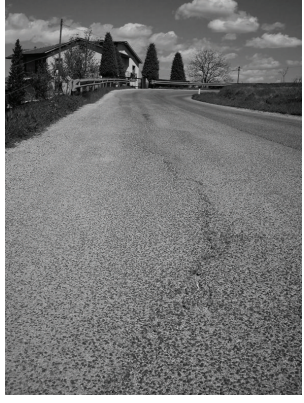

(e)

FIGURE 1: Structural defects investigated in this study: (a) longitudinal and transverse cracks, (b) longitudinal cracks only, (c) transverse cracks only, (d) ramified cracks, and (e) failures. The half road pavement is about $3 \mathrm{~m}$.

causes: unevenness of the subgrade-supporting course; poor workmanship in the placing of layers; poor or uneven compaction; incorrect finishing (water puddles); nonhomogeneous thickness. Deformations due to repeated traffic stresses during road use chiefly originate in the loose granular layers and subgrade of the pavement. They are caused by the use of unsuitable materials, such as granular mixes with crushable aggregates; granular mixes with excess of fines; granular mixes with plastic fines; aggregates of inappropriate grain size; plastic or compressible subgrades.

During road use, bituminous-concrete layers may also contribute to structural degradation of the road surface for the following reasons: presence of crushable aggregates; inappropriate grain size curve; bitumen unsuitable for local climate and traffic conditions; incorrect batching of bitumen; inadequate thickness (undersizing); bituminous concretes that are too stiff with respect to the nonbound underlying layers.

Our paper deals with degradation occurring in flexible pavements of nonurban secondary roads which were investigated with the with the GPR technique $[5,6]$. The GPR offers many advantages, such as speed, low cost, nondestructiveness, minor interference with road traffic, safety, tracing of the causes of degradation, and reliability of results [7]. The study involved statistical analysis of the $1600 \mathrm{MHz}$ radar signal under different conditions of road pavement degradation. The selected approach was based on the assumption that a statistical study on an extended population may yield satisfactory results. Therefore, reliance was made on a large number of scans taken in selected sites whose road section was known from boreholes, digs, and data supplied by management entities. The reliability of the method was based on a comparison of two different conditions (damaged and undamaged) of the same site with similar geometric and geotechnical characteristics.

\section{GPR Experimental Approach}

The study was intended to apply the GPR technique to the assessment of degraded road pavements built in cutting sections and to find correlations between the following variables: types of degradation, depth of cutting sections, and traffic load and GPR data [8]. The study was restricted
TABLe 1: Values of $\alpha_{d}, \alpha_{u}$, and $\Delta \alpha$.

\begin{tabular}{lcccc}
\hline Section & $\alpha_{d}$ & $\alpha_{u}$ & $\Delta \alpha$ & Type \\
\hline (1) LTC-HC-HT & 13.42 & 12.40 & 1.02 & $1 \mathrm{~A}$ \\
(2) LTC-HC-LT & 20.77 & 21.15 & -0.38 & $1 \mathrm{~A}$ \\
(3) LTC-LCu-HT & 27.43 & 28.54 & -1.11 & $1 \mathrm{~A}$ \\
(4) LTC-LCu-LT & 35.72 & 34.91 & 0.81 & $1 \mathrm{~A}$ \\
\hline (5) LCr-HC-HT & 19.37 & 19.82 & -0.45 & $1 \mathrm{~A}$ \\
(6) LCr-HC-LT & 12.47 & 11.47 & 1.00 & $1 \mathrm{~A}$ \\
(7) LCr-LCu-HT & 13.25 & 12.51 & 0.74 & $1 \mathrm{~A}$ \\
(8) LCr-LCu-LT & 11.28 & 12.29 & -1.01 & $1 \mathrm{~A}$ \\
\hline (9) TC-HC-HT & 10.12 & 11.26 & -1.14 & $1 \mathrm{~A}$ \\
(10) TC-HC-LT & 15.58 & 16.28 & -0.07 & $1 \mathrm{~A}$ \\
(11) TC-LCu-HT & 25.56 & 24.72 & 0.84 & $1 \mathrm{~A}$ \\
(12) TC-LCu-LT & 18.34 & 17.14 & 1.20 & $1 \mathrm{~A}$ \\
\hline (13) RC-HC-HT & 10.48 & 7.12 & 3.36 & $2 \mathrm{~B}$ \\
(14) RC-HC-LT & 14.38 & 11.27 & 3.11 & $2 \mathrm{~B}$ \\
(15) RC-LCu-HT & 18.36 & 22.68 & -4.32 & $3 \mathrm{~B}$ \\
(16) RC-LCu-LT & 16.25 & 23.72 & -7.47 & $3 \mathrm{~B}$ \\
\hline (17) F-HC-HT & 13.95 & 10.47 & 3.48 & 2B \\
(18) F-HC-LT & 26.27 & 22.12 & 4.15 & 2B \\
(19) F-LCu-HT & 15.45 & 18.32 & -2.87 & $3 \mathrm{~B}$ \\
(20) F-LCu-LT & 21.75 & 27.49 & -5.74 & $3 \mathrm{~B}$ \\
\hline
\end{tabular}

to 5 different types of structural defects (impaired loadbearing properties) (Figure 1 and Table 1): longitudinal and transverse cracks (LTC), longitudinal cracks only (LCr), transverse cracks only (TC), and ramified cracks (RC) and failures (F). All of the investigated defects were selected to represent worst-case conditions (high degradation): (i) for linear cracks - cracks with a length exceeding $5 \mathrm{~m}$ and a width exceeding $5 \mathrm{~mm}$; (ii) for failures-surface areas of more than $5 \mathrm{~m}^{2}$.

To assess the impact of cutting depth and traffic load, the study took into consideration pavements built in high cuttings ( $\mathrm{HC}$ - depth above $4 \mathrm{~m}$ ) and low cuttings ( $\mathrm{LCu}-$ depth below $2 \mathrm{~m}$ ), as well as surfaces exposed to heavy traffic (HT) with an average daily traffic of $>4000$ and to low traffic (LT) with an average daily traffic of $<1000$. The study covered 40 sites, representing different combinations 


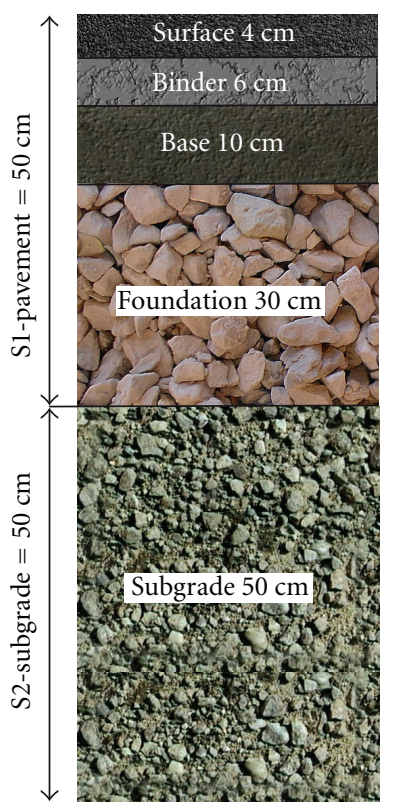

FIGURE 2: Scheme of the investigated road section.

of the investigated variables. The sites were selected on a $20-\mathrm{km}$ long nonurban secondary road in the area of L'Aquila (central Italy). The area under study lies at an altitude of about $800 \mathrm{~m}$ above sea level and has a long and cold winter season.

All of the investigated road superstructures consist of flexible pavements and practically equivalent courses, with the following thickness values: granular-mix foundation course $30 \mathrm{~cm}$; bitumen-mix base course $10 \mathrm{~cm}$; binder course $6 \mathrm{~cm}$ and surface course $4 \mathrm{~cm}$, both in bituminous concrete. Thus, the superstructures have an overall average thickness of $50 \mathrm{~cm}$ (Figure 2).

GPR surveys were conducted to quantitatively analyse signal attenuation versus time curves (power curves and power diagram) with the goal to identify the likely cause of road pavement degradation. In a considerable number of sites, the statistical comparison of damaged and undamaged zones through the absorption coefficient of power curves shows that surface investigations of road pavements are consistent with power curve analyses.

The absorption coefficient is the angle between the horizontal line and the linear regression line, which is generated under the criterion of maximisation of the $R^{2}$ correlation coefficient for the absorbed energy versus time curve (power diagram). The mean power absorption was calculated on a $1.5 \mathrm{~m}$-wide road section, previously selected on the basis of radar signal characteristics. For each radar section, the ratio between at least two zones with extreme surface conservation conditions (damaged versus undamaged) was investigated. The power diagram was obtained (by portions of radar section) by applying mean value removal filters along the scanning direction (background removal filter, which removes ringing and main bang effects, ie, horizontal disturbances due to decoupling between the antenna and the ground).

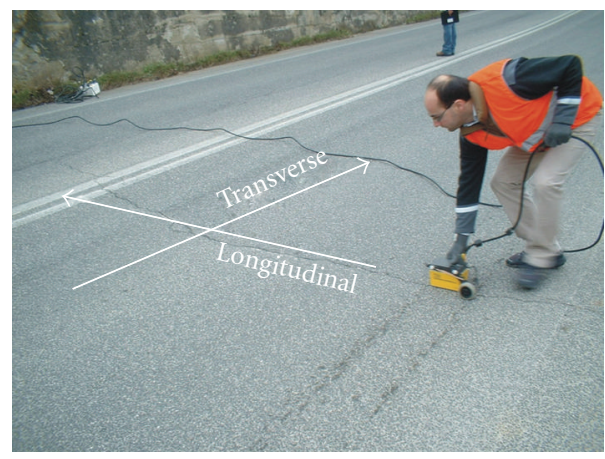

FIGURE 3: GPR acquisition in the road pavement site with $1600 \mathrm{MHz}$ antenna.

\section{GPR Instrumentation}

The GPR emits radio frequency signals in the typical range of $600-2000 \mathrm{MHz}$ for road media and records the echoes which are radiated by the objects contained in the medium or by different types of layers making up the road. GPR reflections arise whenever there is an electromagnetic impedance contrast between the different layers [9-12] of the investigated road pavements or when the media contain a large inhomogeneity, such as a technological pipe. The GPR thus enables to investigate electromagnetic and associated physical properties of pavement layers can be investigated, for example, asphalt characteristics [13], water content [1416], and void ratio [14] of road pavements.

In this study, use was made of an antenna array with a nominal frequency of $1600 \mathrm{MHz}$ (manufactured by Ingegneria dei Sistemi, Pisa, Italy) and a sampling rate of 1024 samples per scan, giving fairly reliable results down to depth of $1.5 \mathrm{~m}$. This choice was based on the consideration that traffic stresses on road cuttings reach a maximum depth of about $1.5 \mathrm{~m}$ and are more pronounced in the first $50 \mathrm{~cm}$ of the superstructure.

\section{Experimental Contour Conditions and GPR Data Acquisition}

In the selected 40 sites $1600 \mathrm{MHz}$ and $600 \mathrm{MHz}$ scans were taken over a total acquisition of $1600 \mathrm{~m}$. For each site, two orthogonal sections were obtained (longitudinal and transverse to the road pavement) (Figure 3). The GPR sections obtained with the two antennas, were processed with time- and space-domain filterings so as to gain greater understanding of the road pavement stratigraphy and of the buried technological networks (Figures 4 and 5).

The experimental contour conditions were as follows.

(i) A negligible water content in the road layers, because (i) the GPR scans were carried out during a dry period and (ii) the sites do not show appreciable groundwater seepage into the geological bedrock. So, water did not interfere with GPR section and with power diagram analysis. 


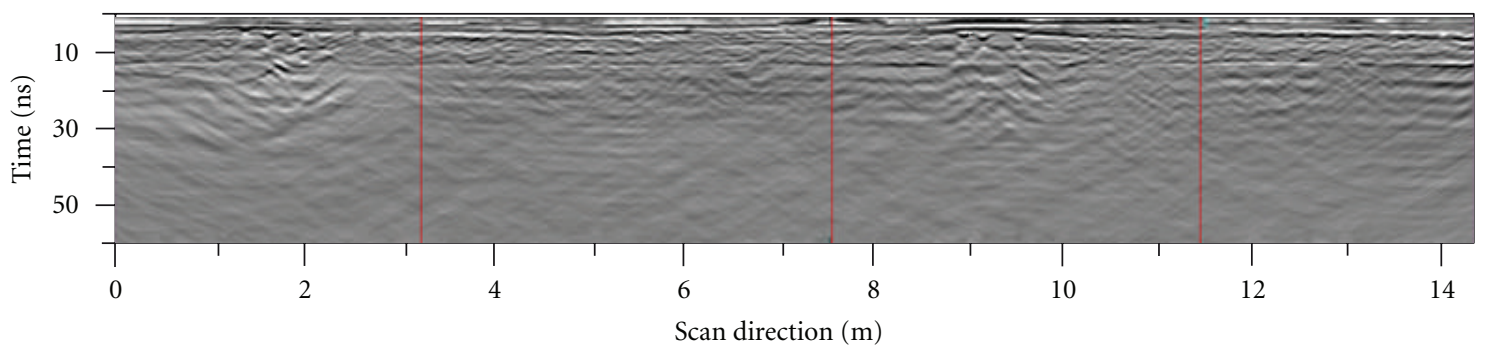

(a)

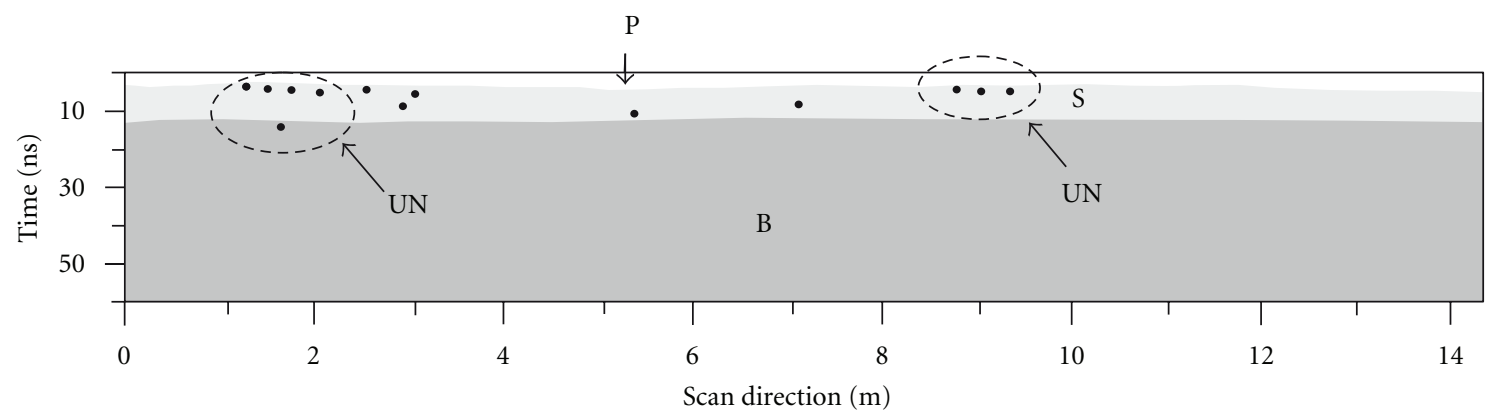

- Point anomaly

(b)

Figure 4: $600 \mathrm{MHz}$ migrated radar longitudinal section (a) and its line drawing (b) of the undamaged road section reported in Figure 3; different road layers (P: pavement; S: subgrade; B: bedrock) and buried underground network (UN) are drawn.

(ii) The characteristics of the geotechnical bedrock, which is made up of stratified calcarenites, were homogeneous down to $1.5 \mathrm{~m}$ (maximum depth of investigation of the $1600 \mathrm{MHz}$ antenna).

The differences between the $1600 \mathrm{MHz}$ radar sections of the damaged (Figure 6) and undamaged (Figure 5) road sections permitted to trace the causes of the investigated types of degradation in the damaged road section versus cutting depth and traffic load. The power diagram was built by analysing only the $1600 \mathrm{MHz}$ radar sections of homogeneous undamaged road pavements and using the background removal filter.

Boundary conditions, which affect the choice of the absorption coefficient, depend on the type of the radar signal that is acquired. Based on this choice, the ratio between the two coefficients of the undamaged and damaged zones nullifies most of the existing variables, bringing about instrumental and operating errors. Therefore, the following conditions were supposed to be minimum or equal to zero: (i) environmental conditions at the surface (e.g., temperature, humidity); (ii) subsurface conditions, considering that the radar section was examined beforehand to exclude zones with localised and linear discontinuities from the subsequent analysis; (iii) operating conditions of the instrument upon the acquisition of radar scans. Data acquisition was based on a rate of 1024 samples per scan.

\section{Data Processing and Interpretation}

As is known, in the Maxwell's equations, the attenuation constant expresses the amount of energy absorbed by the crossed media and, in our case, the road layers [5]. The higher the voids ratio (i.e., porosity: voids volume/total volume) of the investigated material, the higher the radar signal attenuation and the higher the attenuation constant [17-19]. Hence, determining the attenuation constant can give a good assessment of the depth of penetration of the signal.

The Skin Depth is defined as the thickness of the investigated medium, within which a pulse of unit amplitude diminishes to $1 / \mathrm{e}$ (where $\mathrm{e}$ is the base of the natural logarithm) as a consequence of the attenuation characteristics of the same medium. Moreover, the radar signal can be considered to be practically absorbed when the depth of investigation is more than three times the skin-depth of the medium [20].

To assess structural defects in terms of loss of loadbearing capacity, the scans were concentrated on a maximum depth of $1.0 \mathrm{~m}$ from the road surface. This choice was 

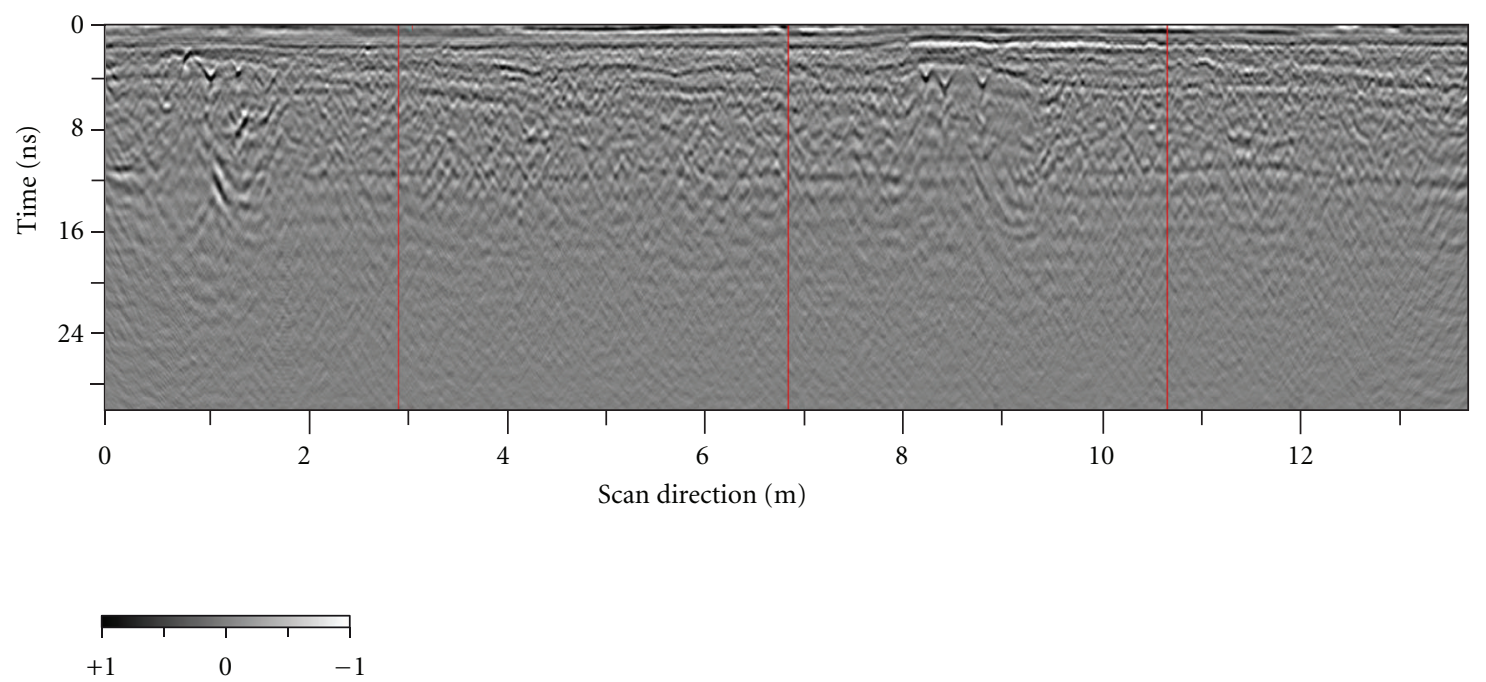

(a)

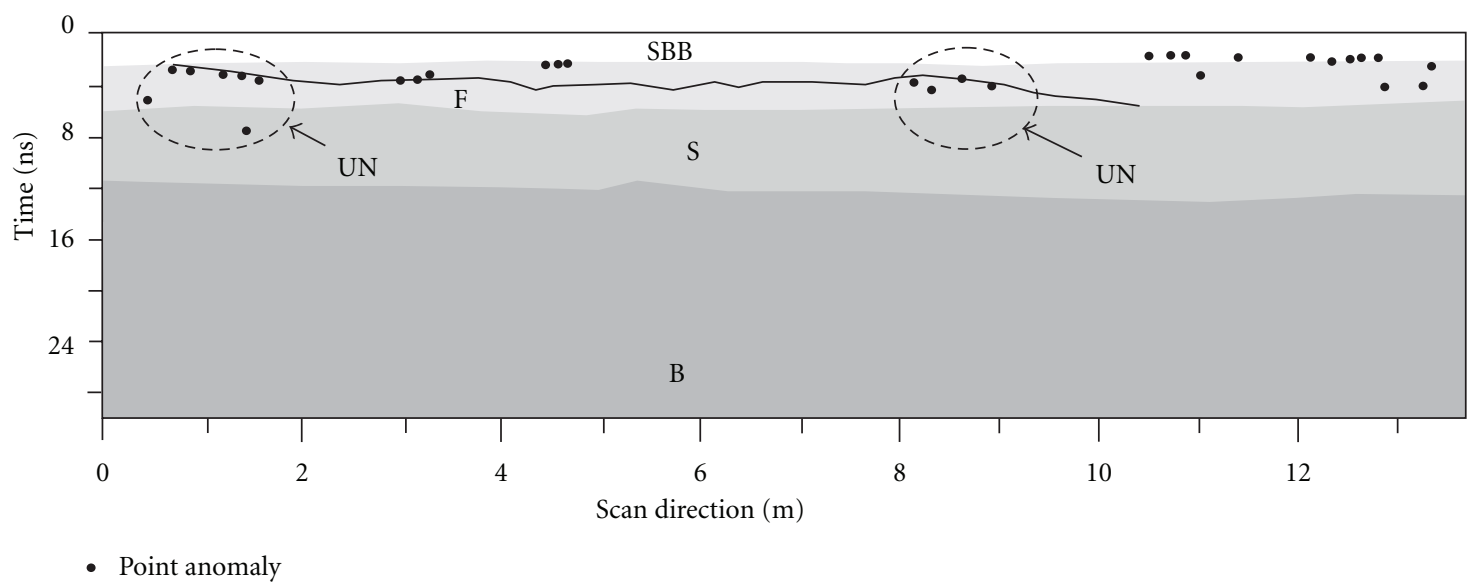

(b)

FIGURE 5: $1600 \mathrm{MHz}$ migrated radar longitudinal section (a) and its line drawing (b) of the undamaged road section reported in Figure 3; different road layers (SBB: surface-binder-base; F: foundation; S: subgrade; B: bedrock) and buried underground network (UN) are drawn; the corresponding power diagram is reported in Figure 9.

made because traffic-generated stresses on roads exert their effects at a depth that does not exceed $1.5 \mathrm{~m}$ on average; moreover, they are more pronounced in the first $50 \mathrm{~cm}$ of the superstructure and they propagate, with still clear and easily detectable effects, for another $50 \mathrm{~cm}$. Therefore, the reference road section was modeled in two portions: the first $(50 \mathrm{~cm}-$ S1) representing the superstructure, and the second (another $50 \mathrm{~cm}$-S2) representing the portion of the subgrade soil still clearly displaying traffic effects (Figure 2). The model was consistent with the $1600 \mathrm{MHz}$ antenna resolution. Under another fundamental assumption of the model, the layers S1 (pavement) and S2 (subbase soil) were considered to be averagely homogeneous. This assumption does not faithfully reflect reality (especially for layer S1), since the characteristics of the materials used in road superstructures (e.g., grain size, specific gravity, and shape) are extremely variable.

The power diagram was obtained for $1.5 \mathrm{~m}$-wide square portions of radar section without underground pipes by applying mean value removal filters along the scanning direction (background removal filter, which removes ringing and main bang effects, i.e., horizontal disturbances due to the decoupling between the antenna and the ground).

The rectified power analysis (via the IDSGred software) [21] graphically showed the linearization with regression analysis of the mean power curve (power in $\mathrm{dB}$ versus time in ns). We analysed the energy absorbed by the portion of the soil of the cutting section lying at a depth of 50-100 cm (S2 in Figure 2). The interpretation of the rectified power diagram made it possible to determine the absorption angle $\alpha^{\prime}$, which is the angle between the horizontal line and the linear regression one generated under the criterion of maximisation of the $R^{2}$ correlation coefficient for the absorbed energy versus time curve (power diagram) (Figure 7).

In all the studied sites, the e.m. propagation velocity over the selected timescale was set to $10 \mathrm{~cm} / \mathrm{ns}$ as a typical value for calcareous road layers (pavement and subgrade) 

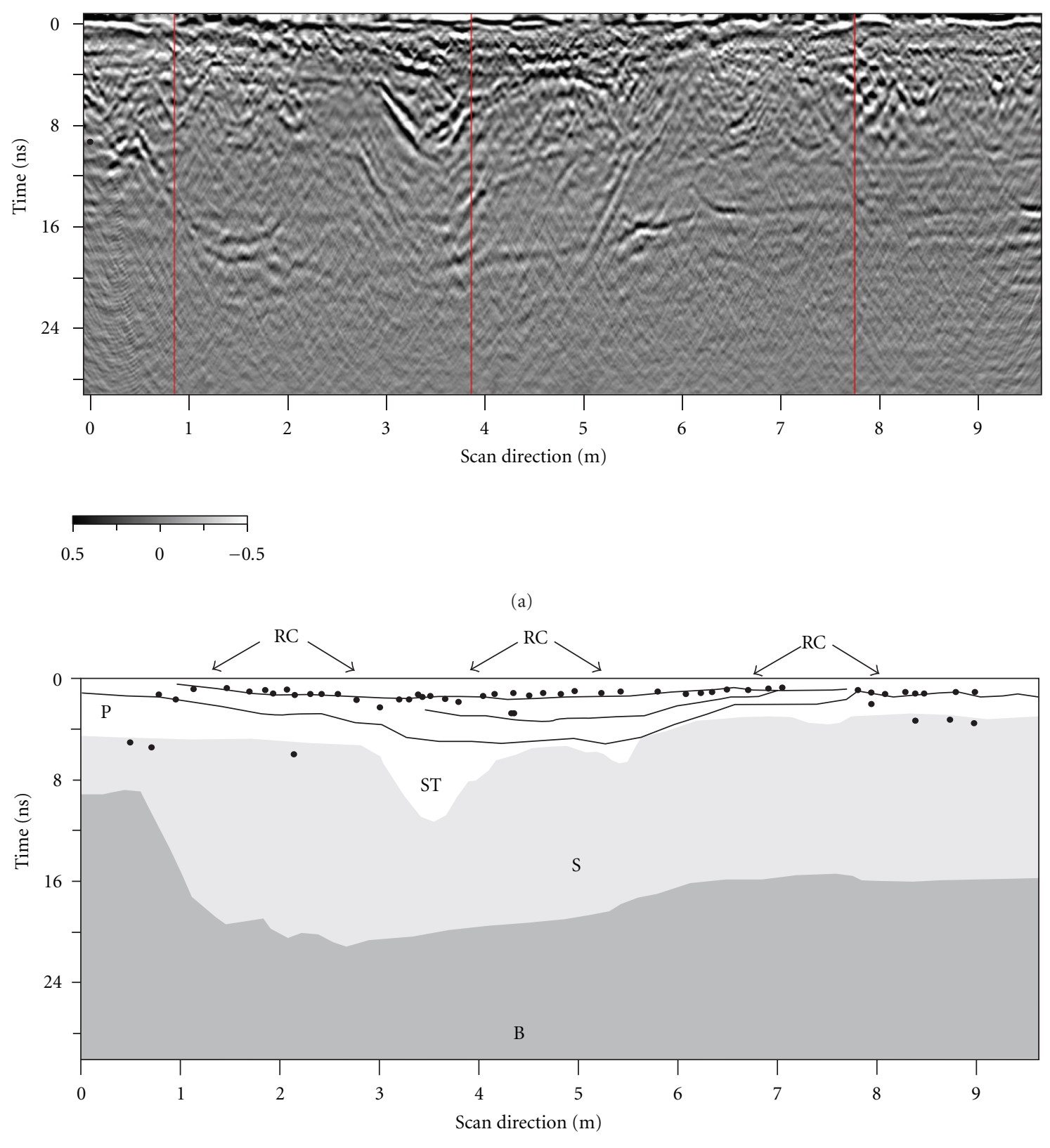

- Point anomaly

(b)

FIGURE 6: Damaged road section due to pavement settlement (ST) and ramified cracks (RC). $1600 \mathrm{MHz}$ migrated radar section (a) and its line drawing (b); different road layers (P: pavement; S: subgrade; B: bedrock) are drawn; the corresponding power diagram is reported in Figure 10.

and bedrock [22] for sites with the same geometrical and geotechnical characteristics. Therefore, a direct proportionality was assumed between the absorption angle $\left(\alpha^{\prime}\right)$, calculated on the received power, and the absorption coefficient $(\alpha)$ thus measuring the absorption coefficient $(\alpha)$ of the entire road pavement site and not those of each road layer.

It is worth recalling that GPR scans with the $1600 \mathrm{MHz}$ antenna were taken on two $1.5 \mathrm{~m}$-long road sections, one damaged and the other undamaged, and that the selected undamaged road section was free of anomalies potentially biasing the comparison with the damaged road section. The radargrams of the two road sections (damaged and undamaged) were included in the same graph, so as to highlight their differences. The black diagrams refer to the damaged road section, while the grey ones refer to the undamaged one.

If the absorption angles of damaged $\left(\alpha_{d}\right)$ and undamaged $\left(\alpha_{u}\right)$ road sections are compared, and if the difference between their values $\left(\Delta \alpha=\alpha_{d}-\alpha_{u}\right)$ tends to zero, then the 


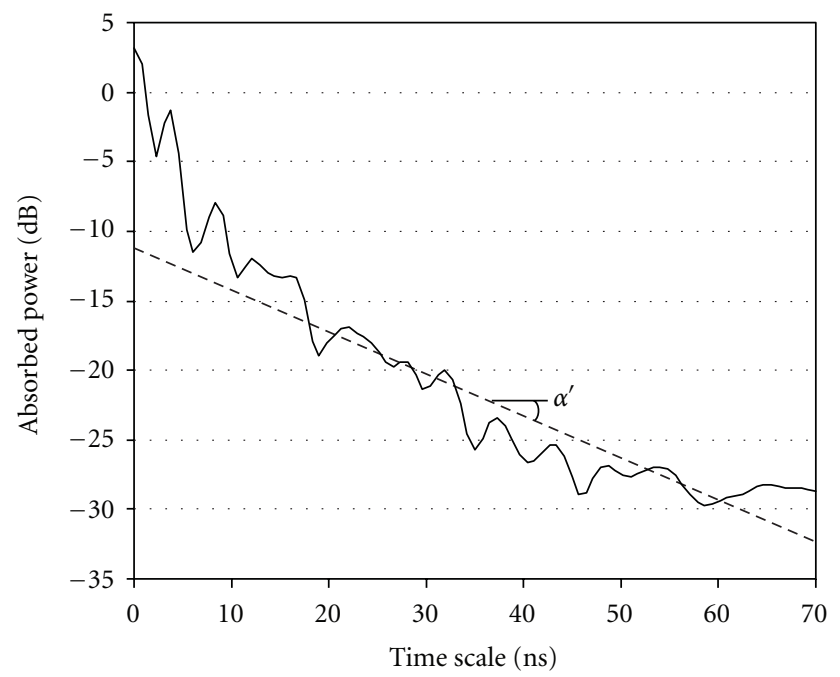

Figure 7: Power diagram with the absorption angle $\left(\alpha^{\prime}\right)$.

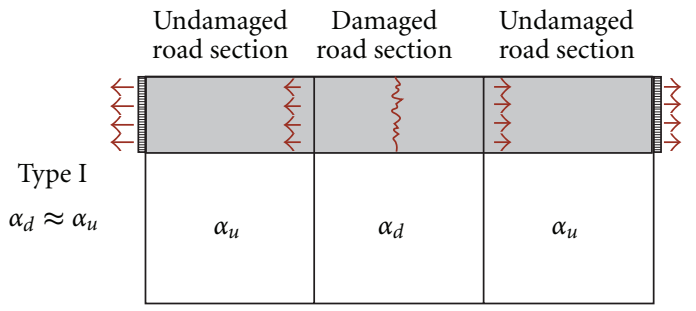

(a)

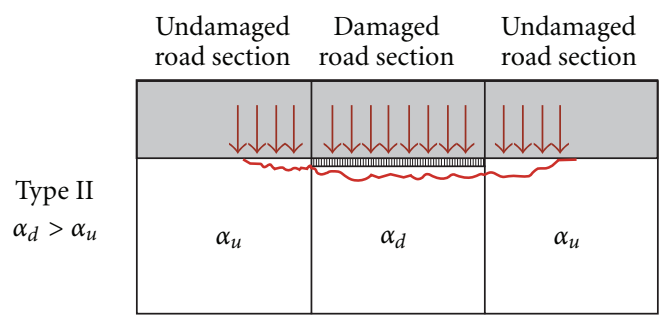

(b)

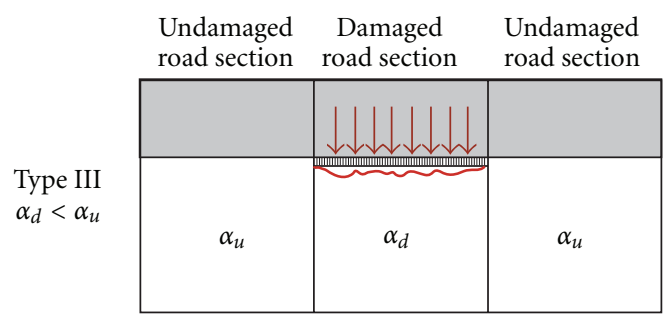

(c)

FIGURE 8: The three types of road section degradation.

likely cause of degradation is fatigue or thermal shrinkage due to horizontal tensile stresses developing in layer S1 of the road pavement (Figure 8(a)). Indeed, in this instance, the energy curves in layer S2 almost coincide. This means that the subgrade soils of the two road sections (damaged and undamaged) have the same density and are not undergoing compaction (Figure 9).
Conversely, if $\Delta \alpha \neq 0$, then the factor triggering road pavement degradation is the different compactness of the soil (portion of the cutting section soil contained in layer S2) due to vehicular traffic load. In this particular case, a further distinction should be made between the case where $\Delta \alpha>0$ and the case where $\Delta \alpha<0$.

In the first case, the energy curves of the damaged zones (black) have lower energy content than the undamaged ones (grey). This means that the damaged zones are more compact than the undamaged ones. More precisely, the energy curves of the damaged zones (black) lie below the undamaged ones (grey), confirming that a lower level of absorbed energy corresponds to a lower voids index (Figure 10). So, the process of degradation is no longer active in the damaged zones; by contrast, the undamaged zones will tend, over time, to become as dense as the damaged ones. Hence, the degradation will tend not to remain confined to the damaged zones but to migrate to the nearby zones (Figure $8(\mathrm{~b})$ ).

In the second case, the energy curves of the damaged zones (black) display higher energy content than the undamaged ones (grey). In other words, the damaged zones are less compact than the undamaged ones. More precisely, the energy curves of the damaged zones (black) lie above those of the undamaged zones (grey), confirming that a higher level of absorbed energy corresponds to a higher voids index (Figure 11). Thus, the process of degradation is still active in the damaged zone and will continue to be so until reaching the density level of the undamaged zone. Hence, the degradation will remain confined to the damaged zone (Figure 8(c)).

\section{Results and Discussion}

It should be pointed out that the accuracy of interpretation of the $1600 \mathrm{MHz}$ sections depends on the resolution of the antenna and on the sampling rate of the radar signal (about $1 \mathrm{~cm}$ and 1024 samples per scan, resp.). Moreover, the resolution of the power diagrams is $1 \mathrm{~dB}$ for signal absorption ( $y$-axis) and $4 \mathrm{~cm}$ for depth from road level $(x-$ axis). This approach thus offers such a high resolution as to permit the scanning of surface areas of up to $1 \mathrm{~cm}^{2}$ in a $1.5 \mathrm{~m}$-thick road pavement or $1.5 \mathrm{~m}$-deep cutting section (Figures 9, 10 and 11).

The power diagram analysis enabled to determine signal absorption angles in both damaged $\left(\alpha_{d}\right)$ and undamaged $\left(\alpha_{u}\right)$ zones and their variations $(\Delta \alpha)$. Each survey has a code identifying the type of degradation (LTC, LCr, TC, RC, and F), the cutting depth ( $\mathrm{HC}, \mathrm{LCu})$, and the traffic load (HT, LT) (Table 1). Based on the analysis of the power diagrams, the types of degradation were divided into two categories: (i) crack-type degradation (LTC, LCr, and TC), identified as CAT. I; (ii) extensive degradation ( $\mathrm{RC}$ and F), identified as CAT. II, as shown in Table 2.

In detail, CAT. I damages gave $\Delta \alpha$ values in the range of $-2<\Delta \alpha<+2$. In Table 1 , these types of damage are denoted with $1 \mathrm{~A}$, as specified in Table 3 . It can be stated that cracktype degradation (CAT. I) is usually generated by horizontal tensile stresses which are due to fatigue or thermal shrinkage, 


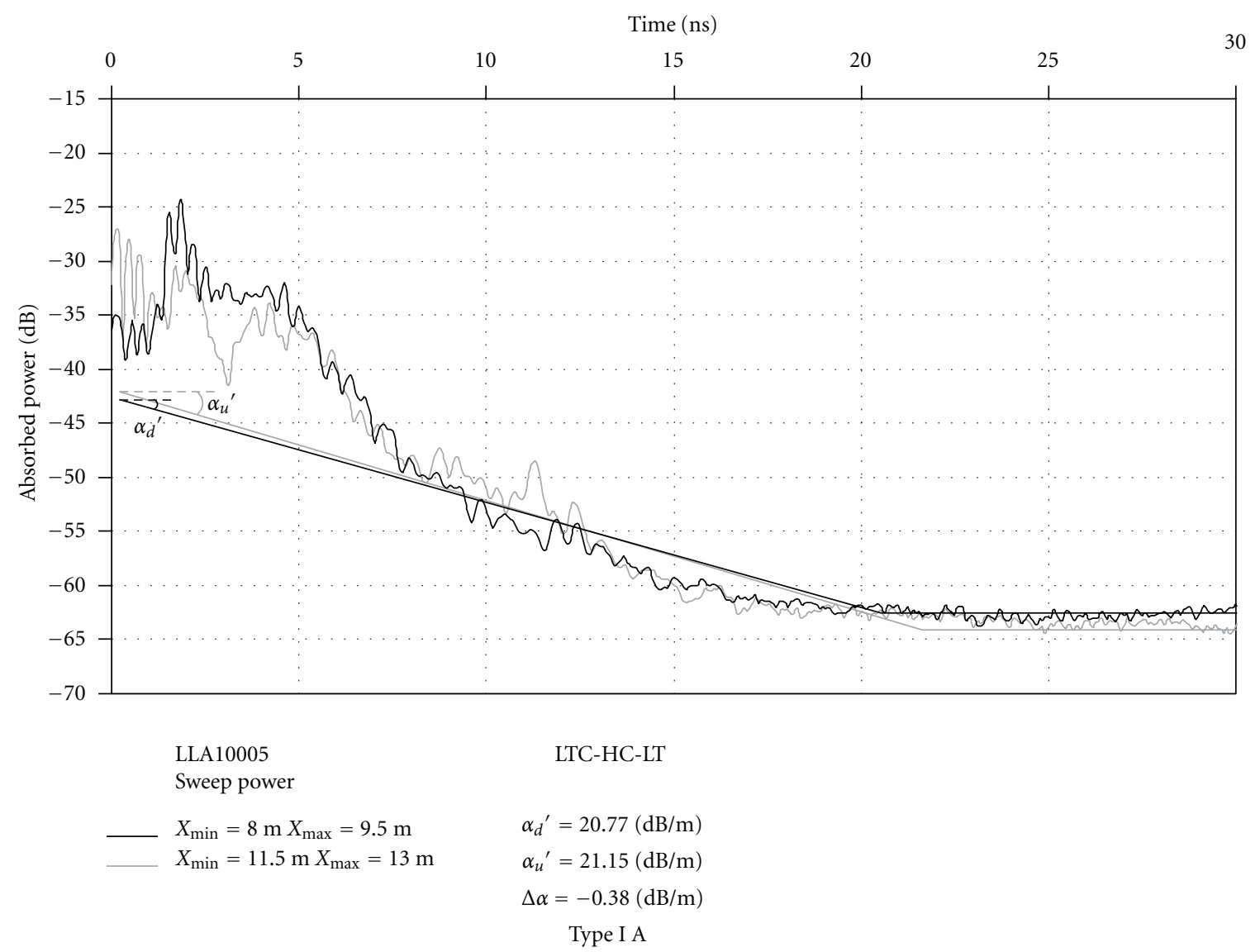

FIgURE 9: Power diagram (case $\Delta \alpha \cong 0$ ) of the $1600 \mathrm{MHz}$ section reported in Figure 5.

TABle 2: Classification of road pavement degradation into two categories.

\begin{tabular}{ll}
\hline Category & Type of Degradation \\
\hline \multirow{2}{*}{ CAT. I } & LTC-longitudinal and transversal cracks \\
& LCr-longitudinal cracks only \\
& TC-transversal cracks only \\
\hline \multirow{2}{*}{ CAT. II } & RC-ramified cracks \\
& F-failures \\
\hline
\end{tabular}

chiefly affecting the shallow layers of the road pavement (type I: Figures 8(a) and 9).

This is possible above all in roads which are built in lowand deep-cutting sections. In this case, fatigue rupture of bituminous-concrete layers is the dominant phenomenon, because (i) the load-bearing road courses are undersized with respect to the actual traffic load; or (ii) bituminousconcrete layers are too stiff with respect to the too deformable underlying layers. Cracks may also originate from thermal shrinkage of shallow bituminous-concrete pavement layers, since the investigated roads are located in fairly cold areas. So it can be inferred that subgrade soils (down to a depth of $1.5 \mathrm{~m}$ ) are not in this case responsible for crack-type degradation.
TABle 3: Types of road pavement degradation with respect to $\alpha_{d}$ and $\alpha_{u}$.

\begin{tabular}{lccc}
\hline & Type I & Type II & Type III \\
\hline Category & $\alpha_{d} \cong \alpha_{u}$ & $\alpha_{d}>\alpha_{u}$ & $\alpha_{d}<\alpha_{u}$ \\
CAT. I & $1 \mathrm{~A}$ & $2 \mathrm{~A}$ & $3 \mathrm{~A}$ \\
CAT. II & $1 \mathrm{~B}$ & $2 \mathrm{~B}$ & $3 \mathrm{~B}$ \\
\hline
\end{tabular}

Additional causes of CAT. I cracks include poor workmanship during superstructure construction and, in particular, unevenness of the subgrade-supporting course; nonhomogeneous thickness of the various layers; inadequate placing of additional layers of superstructure after work interruption.

This does not rule out that cracks detected in the investigated cases may arise from a combination of the above-described factors, whose impact may be different, depending on whether they are concurrent, combined, or cumulated.

With regard to CAT. II degradation, the values were in the range of $12<\Delta \alpha<+8$ range.

In Table 1, these types of degradation are denoted with $2 \mathrm{~B}$ and $3 \mathrm{~B}$ (Table 3 ). Results suggest that extensive degradation (CAT. II) originates from variations in the subgrade soil density induced by vehicular traffic. In particular, if 


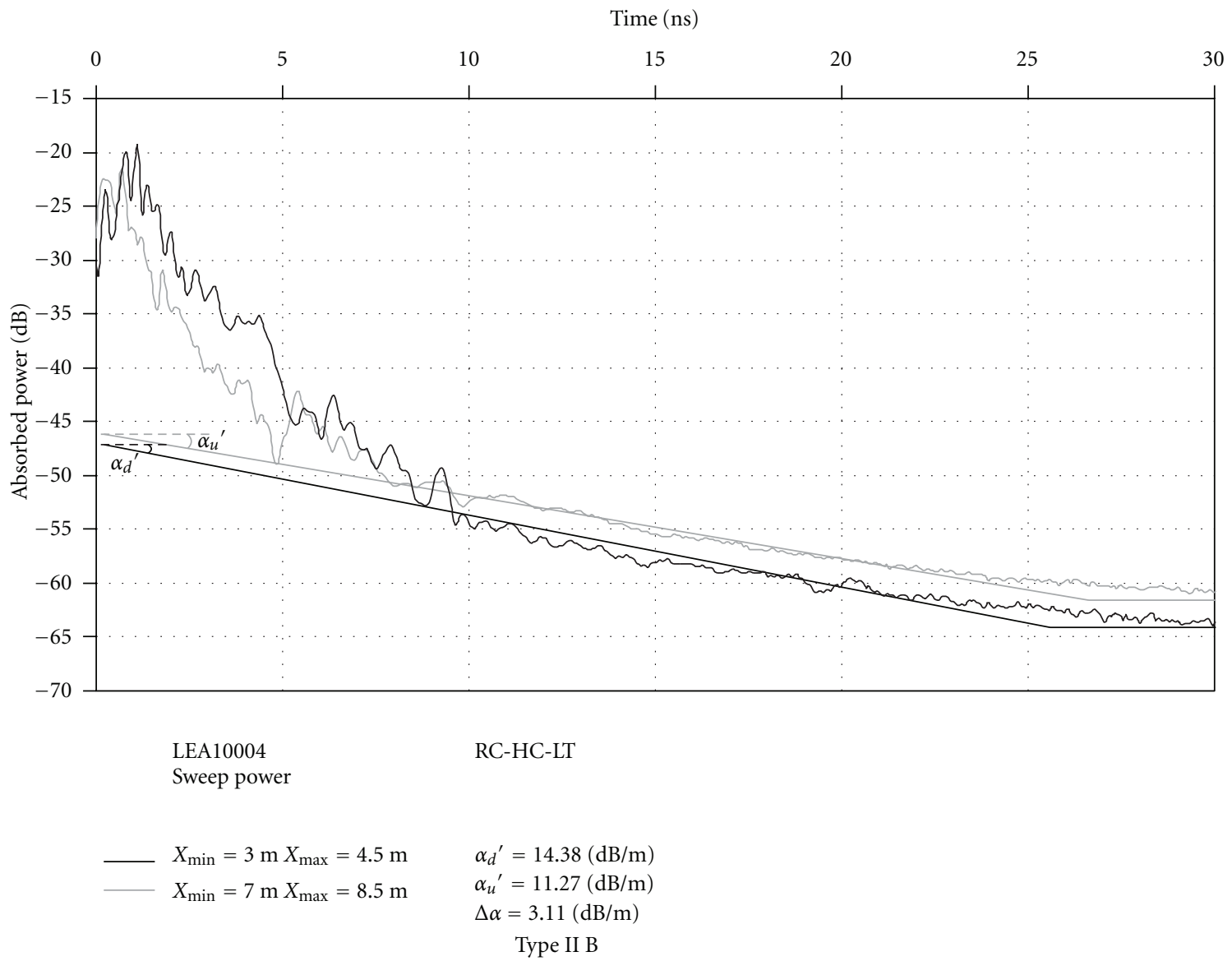

Figure 10: Power diagram (case $\Delta \alpha>0$ ) of the $1600 \mathrm{MHz}$ section reported in Figure 6.

$\alpha_{d}>\alpha_{u}$, the damage will tend to extend to nearby zones (Type II: Figures 8(b) and 10). By contrast, if $\alpha_{d}<\alpha_{u}$, the damage will remain confined to the already damaged zone (Type III: Figures 8(c) and 11). Moreover, in high cuttings (HC), the highest values of $\Delta \alpha$ have all a positive sign (2B). This is tantamount to saying that compaction problems are not limited to layers lying in the proximity of the subgrade, but that they also affect wider subgrade surfaces. In this case, the degradation causes are ascribable to poor soil compaction during subgrade-supporting course preparation or inadequate remediation of the subgrade soil.

For low cuttings $(\mathrm{LCu})$, the highest values of $\Delta \alpha$ have all a negative sign (3B). This means that compaction problems are limited to the layers lying in the proximity of the subgrade, and that they will remain confined to the already damaged zones. In this case, degradation is caused by nonhomogeneous soils (with portions of unsuitable materials, e.g., crushable or compressible materials) or by an unevenly compacted road surface.

In both high cuttings (2B) and low cuttings (3B), degradation of CAT. II road sections, may arise as follows: (i) too stiff bituminous-concrete layers with respect to too deformable underlying layers; (ii) fatigue rupture of bituminous-concrete layers due to supporting layers that are undersized with respect to actual traffic load and, above all, (iii) the subgrade course load-bearing capacity.

\section{Conclusions}

A $1600 \mathrm{MHz}$ radar investigation was conducted on degraded road pavements built in cutting sections. The power curves (power in $\mathrm{dB}$ versus time in ns) were analysed through the rectified power method and subsequently the absorption coefficient $\alpha$ was determined. The power absorption was calculated on a $1.5 \mathrm{~m}$-wide road section, previously selected on the basis of radar signal. For each radar section, the ratio between at least two zones with extreme surface conservation conditions (damaged versus undamaged) was investigated.

A discrimination was made between causes of deep origin, such as inadequate nature or poor compaction of the soil of the cutting section and causes of shallow origin (shallow layers of the superstructure) due to strong horizontal tensile stresses, generated by fatigue or thermal shrinkage and developing inside the road pavement. So if the absorption angles of damaged and undamaged road sections are similar, then the likely cause of degradation is fatigue or thermal shrinkage; if they are not, then the factor triggering of road degradation is the different compactness of 


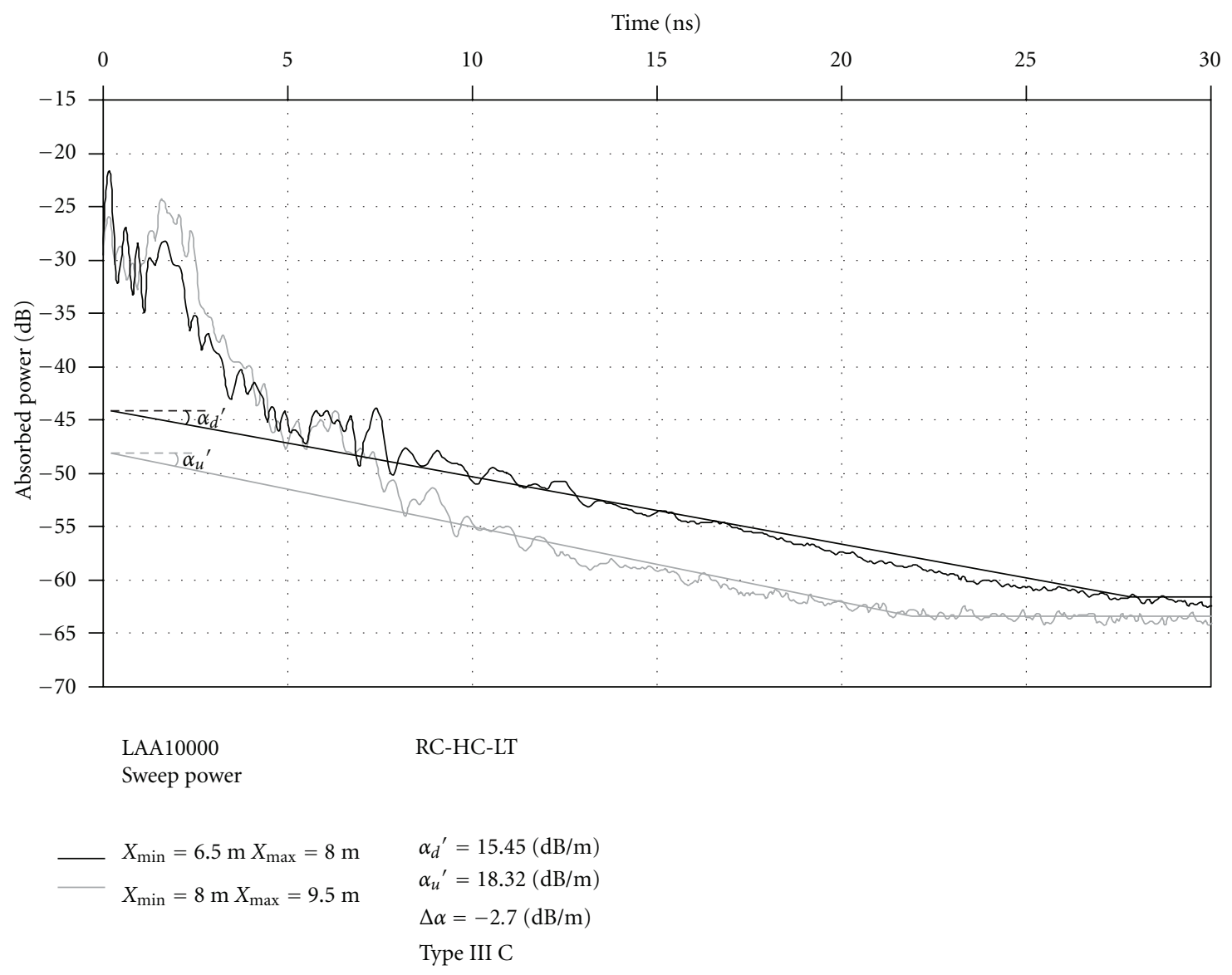

Figure 11: Power diagram (case $\Delta \alpha<0$ ).

the soil due to vehicular traffic load. If GPR signal variations indicate that some subgrade zones have been inadequately compacted $(\Delta \alpha \neq 0)$, then extensive degradation may be inferred (ramified cracks and failures) and the testing will be successful.

\section{Acknowledgments}

The authors are indebted to Marco Scozzafava, for stimulating discussions about the GPR technique and for his considerable help during the GPR survey, and to anonymous referees and Mrinal Sen whose comments significantly improved the first version of the paper.

\section{References}

[1] SITEB, "Manutenzione delle pavimentazioni stradali," Emmevi grafica, Varese, Italy, 2004.

[2] CNR, "Istruzioni sulla pianificazione della manutenzione stradale," Italian regulation, 1988.

[3] SHRP, "Distress identification manual for the long-term pavement performance project," USA regulation, 1993.

[4] VSS, “Catalogue des degradations," Swiss regulation, 1991.

[5] D. J. Daniels, Surface-Penetrating Radar, The Institution of Electrical Engineers; Short Run Press Ltd, Exeter, UK.
[6] T. Saarenketo and T. Scullion, "Road evaluation with ground penetrating radar," Journal of Applied Geophysics, vol. 43, no. 2-4, pp. 119-138, 2000.

[7] S. Smith and T. Scullion, "Development of groundpenetrating radar equipment for detecting pavement condition for preventive maintenance," Tech. Rep. Project H-104 A, Strategic Highway Research Program, Natural Resources Defense Council, Washington, DC, USA, 1993.

[8] K. R. Maser, "Condition assessment of transportation infrastructure using ground-penetrating radar," Journal of Infrastructure Systems, vol. 2, no. 2, pp. 94-101, 1996.

[9] X. Dérobert, C. Fauchard, P. Côte et al., "Step-frequency radar applied on thin road layers," Journal of Applied Geophysics, vol. 47, no. 3-4, pp. 317-325, 2001.

[10] C. Fauchard, X. Dérobert, J. Cariou, and P. Côte, "GPR performances for thickness calibration on road test sites," NDT and E International, vol. 36, no. 2, pp. 67-75, 2003.

[11] M. O. Gordon, K. Broughton, and M. S. A. Hardy, "The assessment of the value of GPR imaging of flexible pavements," NDT and E International, vol. 31, no. 6, pp. 429-438, 1998.

[12] S. Lahouar and I. L. Al-Qadi, "Automatic detection of multiple pavement layers from GPR data," NDT and E International, vol. 41, no. 2, pp. 69-81, 2008.

[13] I. L. Al-Qadi, S. Lahouar, and A. Loulizi, "In situ measurements of hot-mix asphalt dielectric properties," NDT and E International, vol. 34, no. 6, pp. 427-434, 2001. 
[14] A. Benedetto and S. Pensa, "Indirect diagnosis of pavement structural damages using surface GPR reflection techniques," Journal of Applied Geophysics, vol. 62, no. 2, pp. 107-123, 2007.

[15] K. Grote, S. Hubbard, J. Harvey, and Y. Rubin, "Evaluation of infiltration in layered pavements using surface GPR reflection techniques," Journal of Applied Geophysics, vol. 57, no. 2, pp. 129-153, 2005.

[16] M. B. Kowalsky, S. Finsterle, and Y. Rubin, "Estimating flow parameter distributions using ground-penetrating radar and hydrological measurements during transient flow in the vadose zone," Advances in Water Resources, vol. 27, no. 6, pp. 583-599, 2004.

[17] J. L. Davis and A. P. Annan, "Ground-penetrating radar for high-resolution mapping of soil and rock stratigraphy," Geophysical Prospecting, vol. 37, no. 5, pp. 531-551, 1989.

[18] G. C. Topp, J. L. Davis, and A. P. Annan, "Electromagnetic determination of soil water content: measurements in coaxial transmission lines," Water Resources Research, vol. 16, no. 3, pp. 574-582, 1980.

[19] A. Turesson, "Water content and porosity estimated from ground-penetrating radar and resistivity," Journal of Applied Geophysics, vol. 58, no. 2, pp. 99-111, 2006.

[20] A. R. Sena, P. L. Stoffa, and M. K. Sen, "Split-step Fourier migration of GPR data in lossy media," Geophysics, vol. 71, no. 4, pp. K77-K91, 2006.

[21] Ingegneria dei Sistemi, “IDSGred3D User manual. 20," 2000, Pisa, Italy.

[22] S. Colagrande, D. Ranalli, M. Scozzafava, and M. Tallini, "GPR investigations on degraded road pavements built on embankments," in Proceedings of the International Conference on Advanced Characterisation of Pavement and Soil Engineering, A. Loizos, T. Scarpas, and I. L. Al-Qadi, Eds., pp. 971-981, Taylor \& Francis Ltd, Athens, Greece, June 2007. 

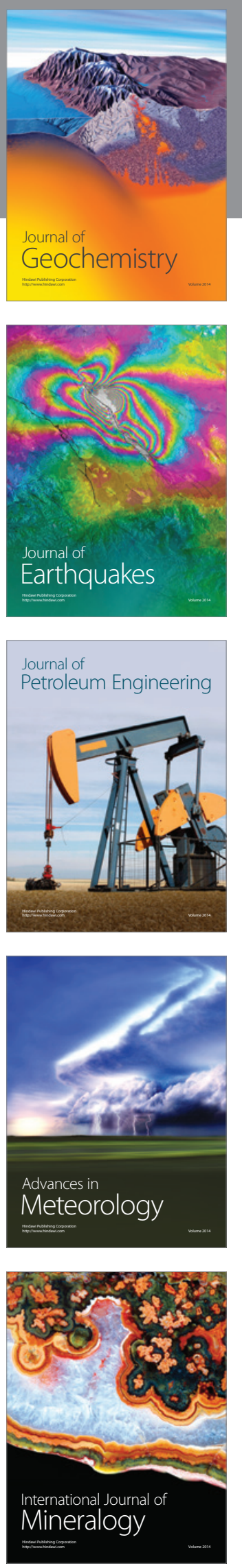
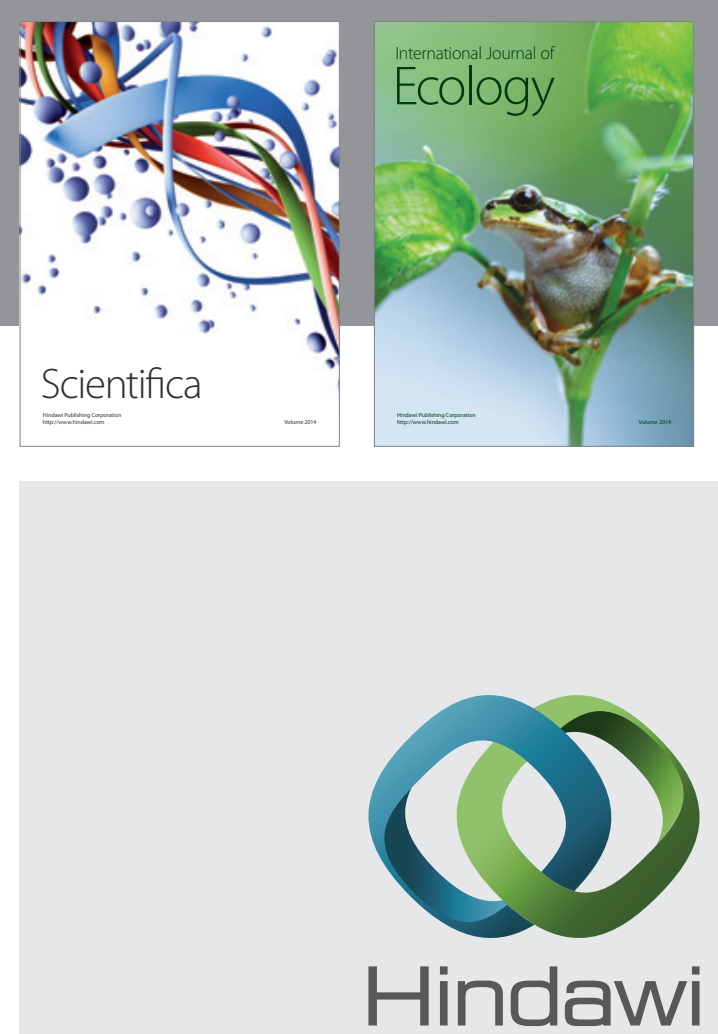

Submit your manuscripts at http://www.hindawi.com
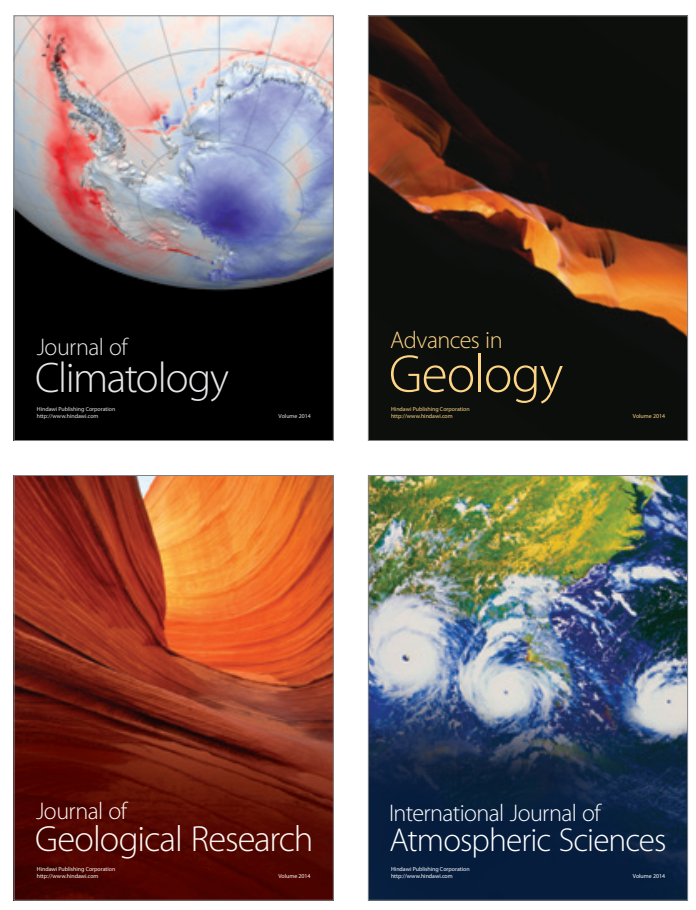
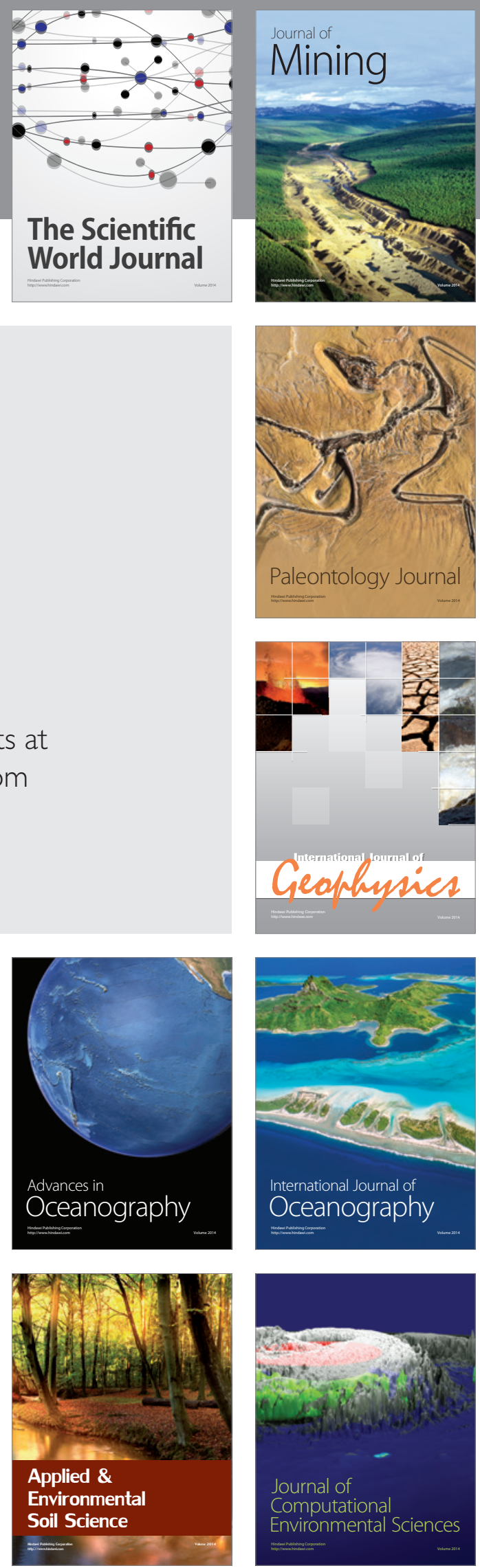